\title{
Possíveis causas de diarréia aguda em crianças de idade pré-escolar
}

\author{
Possible causes of acute diarrhea in preschool age children \\ Posibles causas de diarrea aguda en niños en edad preescolar
}

Kathlyn Cristina Canedo Póvoa

ORCID: https://orcid.org/0000-0002-7834-9312 Universidade Brasil, Brasil

E-mail: tetipovoa@gmail.com

Aline Kelly Wanderley Pereira

ORCID: https://orcid.org/0000-0002-4070-7145 Universidade Brasil, Brasil

E-mail: alinekwp9@gmail.com

Fernanda Ravazi de Morais

ORCID: https://orcid.org/0000-0003-1338-6291 Universidade Brasil, Brasil

E-mail: ferravazi@hotmail.com

Gabriela de Godoy

ORCID: https://orcid.org/0000-0001-6056-1449 Universidade Brasil, Brasil

E-mail: gabi_godoy098@hotmail.com

Aline dos Santos Macedo

ORCID: https://orcid.org/0000-0002-7487-2974 Universidade Brasil, Brasil

E-mail: santosmacedoaline@gmail.com

Matheus Zacharias Vidal

ORCID: https://orcid.org/0000-0002-9821-3113 Universidade Brasil, Brasil

E-mail: mzvzacharias@gmail.com

Leônidas Augusto David Miranda

ORCID: https://orcid.org/0000-0001-6788-1058 Universidade Brasil, Brasil

E-mail: leonidasaugusto1997@gmail.com

Luana Lima Costa

ORCID: https://orcid.org/0000-0001-5439-7312 Universidade Brasil, Brasil

E-mail: luanalimacosta@outlook.com.br

Sinair Mendes Ferreira Junior

ORCID: https://orcid.org/0000-0002-5813-6168 Universidade Brasil, Brasil

E-mail: drsinairjr@gmail.com

Rafaela Caffarena Franco

ORCID: https://orcid.org/0000-0002-9325-9219 Universidade Brasil, Brasil

E-mail: rafah_caffarena@hotmail.com

Melina Aparecida Plastina Cardoso

ORCID: https://orcid.org/0000-0002-0669-1469

Universidade Estadual de Maringá, Brasil

E-mail: melina_cardoso@msn.com

\section{Resumo}

Objetivo: identificar as causas relacionadas à diarreia em crianças pré-escolares e compreender as consequências do quadro a longo prazo. Método: Trata-se de um estudo descritivo, do tipo revisão de literatura, realizado em forma de pesquisa bibliográfica. Resultados: as principais causas relacionadas à diarreia em crianças de idade pré-escolar são: condições sociais, intoxicação alimentar e agentes patogênicos, falta de saneamento básico, ausência de imunização e alergias e intolerâncias alimentares, e suas consequências estão relacionadas com prejuízos no desenvolvimento físico e cognitivo da criança. Conclusão: os objetivos propostos foram atingidos, uma vez que foi possível, através da pesquisa bibliográfica em questão, apontar quais as causas e possíveis consequências de diarreias agudas em crianças. Os achados permitiram identificar condições sociais, intoxicação alimentar e agentes patogênicos, falta de saneamento básico, ausência de imunização e alergias e intolerâncias alimentares como as principais causas, e com isso, enfatiza-se a 
necessidade de estimular medidas profiláticas e enfatizar a participação de pais e responsáveis em programas relacionados à prevenção de possíveis infecções, e estímulo a imunização.

Palavras-chave: Diarréia; Crianças pré-escolares; Causas de diarréia; Consequências de diarréia; Agentes patogênicos.

\begin{abstract}
Objective: to identify the causes related to diarrhea in preschool children and understand the consequences of the condition in the long term. Method: This is a descriptive study, of the literature review type, carried out in the form of a bibliographic research. Results: the main causes related to diarrhea in preschool-age children are: social conditions, food poisoning and pathogens, lack of basic sanitation, lack of immunization and food allergies and intolerances, and their consequences are related to impairments in physical development and cognitive of the child. Conclusion: the proposed objectives were achieved, as it was possible, through the bibliographical research in question, to point out the causes and possible consequences of acute diarrhea in children. The findings allowed the identification of social conditions, food poisoning and pathogens, lack of basic sanitation, lack of immunization and food allergies and intolerances as the main causes, and with this, the need to encourage prophylactic measures and emphasize the participation of parents is emphasized. and responsible for programs related to the prevention of possible infections, and encouragement of immunization.
\end{abstract}

Keywords: Diarrhea; Preschool children; Causes of diarrhea; Consequences of diarrhea; Pathogenic agents.

\title{
Resumen
}

Objetivo: identificar las causas relacionadas con la diarrea en niños en edad preescolar y comprender las consecuencias de la enfermedad a largo plazo. Método: Se trata de un estudio descriptivo, del tipo revisión de la literatura, realizado en forma de investigación bibliográfica. Resultados: las principales causas relacionadas con la diarrea en niños en edad preescolar son: condiciones sociales, intoxicaciones alimentarias y patógenos, falta de saneamiento básico, falta de inmunización y alergias e intolerancias alimentarias, y sus consecuencias están relacionadas con deficiencias en el desarrollo físico y cognitivo del niño. Conclusión: se lograron los objetivos propuestos, ya que fue posible, a través de la investigación bibliográfica en cuestión, señalar las causas y posibles consecuencias de la diarrea aguda en niños. Los hallazgos permitieron identificar las condiciones sociales, intoxicaciones alimentarias y patógenos, falta de saneamiento básico, falta de inmunización y alergias e intolerancias alimentarias como las principales causas, y con ello, se enfatiza la necesidad de incentivar las medidas profilácticas y enfatizar la participación de los padres. y responsable de los programas relacionados con la prevención de posibles infecciones y el fomento de la inmunización.

Palabras clave: Diarrea; Niños de preescolar; Causas de la diarrea; Consecuencias de la diarrea; Agentes patógenos.

\section{Introdução}

Segundo relatório publicado pelo Ministério da Saúde, diarréias agudas são consideradas doenças infecciosas gastrointestinais (Brasil, 2021) caracterizadas por ocorrência de, no mínimo, três episódios em até 24 horas, consistência líquida, aumento de evacuações e duração de até 14 dias. Esse quadro pode ser acompanhado de outros sintomas, como: dor abdominal, cefaléia, febre, náusea, dores de cabeça, dores abdominais, cólicas e sangramento nas fezes.

Crianças em idade pré-escolar, de 2 a 6 anos, caracterizam-se como sendo uma faixa populacional que requer atenção, pois nessa fase há um processo de maturação biológico intenso, que requer cuidados e especificidades, principalmente por parte do responsável. Além disso, é nessa etapa da vida da criança que a alimentação desempenha um papel crítico e decisivo, pois podem acarretar em alguns distúrbios pela adaptação da sua alimentação (Gandra, 1981). Nessa fase, ocorrem muitas diarréias agudas, que proporcionam preocupação aos pais, além de prejuízos à saúde da criança e seu desenvolvimento pleno e integral.

As principais causas relacionadas à diarréia aguda em crianças de idade pré-escolar, assim como em alguns adolescentes e adultos são: condições sociais (saneamento básico e pobreza), agentes patogênicos (bactérias, vírus e parasitas que podem estar presentes em alimentos, água ou objetos contaminados e/ou manipulação inadequada de alimentos), falhas na imunização e intolerâncias e alergias alimentares (intolerâncias à lactose e alergias ao glúten) (Moraes \& Castro, 2014).

O diagnóstico para identificação das principais causas etiológicas é realizado por pesquisas de microorganismos nas fezes, primordiais para que se estabeleçam condições terapêuticas. Vale ressaltar que a coleta deve ser realizada com 2 ou 3 amostras por paciente, antes mesmo da administração de qualquer medicamento indicado por profissional da saúde (Brasil, 2021). 
Algumas consequências são consideradas moderadas, porém, algumas são graves e até irreversíveis como: desnutrição, parada de crescimento, quadros de anemia, algumas deficiências - principalmente nutritivas - e, quando não tratada, podem levar ao óbito (SBP, 2021).

Apesar do assunto ser amplamente discutido e difundido, há ainda algumas lacunas a serem conhecidas e compreendidas, o principal objetivo deste trabalho pauta-se em identificar as causas relacionadas à diarreia em crianças pré-escolares e compreender as consequências do quadro a longo prazo.

\section{Metodologia}

Trata-se de um estudo descritivo, do tipo revisão de literatura, realizado em forma de pesquisa bibliográfica. Segundo Kleina e Rodrigues (2014, p. 155): “A pesquisa bibliográfica organiza e sintetiza de forma crítica as informações já disponíveis sobre o tema estudado, por meio da seleção dos principais autores que pesquisaram e escreveram sobre o assunto".

Dentro de uma pesquisa bibliográfica, o papel do autor é buscar informações relevantes acerca do tema. Tal feito só é alcançado, através da busca, leitura, análise e transcrição dos dados em forma de texto, imagens, gráficos, etc. Neste tipo de pesquisa, admite-se um caráter descritivo do tipo qualitativo (Estrela, 2018).

Em pesquisas descritivas, o papel do autor é levantar, registrar e descrever os fatos observados ou colhidos, sem interferir nos mesmos. Possui ainda, o objetivo de descrever de forma detalhada sobre o fenômeno que circunda a temática escolhida (Prodanov $\&$ de Freitas, 2013).

Os descritores, ou seja, palavras chave utilizadas para fazer realizar a busca foram: "diarréia aguda", "diarréia aguda em crianças", "diarréia aguda em crianças de idade pré-escolar", "causas relacionadas à diarréia aguda em crianças" e “consequências da diarréia aguda", publicados nos períodos de 2000 a 2021.

Os critérios de inclusão utilizados neste estudo foram: artigos completos, disponibilizados na íntegra, nos idiomas inglês e português, publicados no período de 2000 a 2021. Já os critérios de exclusão utilizados durante a busca, foram: artigos duplicados, cartas editoriais e relatos de experiência. As bases científicas utilizadas para a busca foram: Google scholar, PubMed, Lilacs e Scielo. Foi realizada uma leitura minuciosa dos resumos de cada um dos artigos selecionados na íntegra, para que pudessem ser incluídos ou não na discussão deste artigo.

\section{Resultados}

Na presente revisão integrativa, aplicando-se os critérios de inclusão e exclusão, foram encontrados 46 artigos. Após a exclusão de artigos duplicados alcançou-se o total de 40 artigos para a leitura dos resumos e trabalhos na íntegra. Pela leitura, 6 artigos foram excluídos por não apresentarem um ou alguns do(s) descritor(es) previamente selecionados e mencionados no item "Metodologia". Após essa leitura, chegou-se a um total de 34 artigos incluídos na revisão.

Quanto aos demais documentos considerados pertinentes a este estudo, foram encontrados e utilizados um total de 24 artigos, e 10 documentos científicos, todos disponíveis na internet, na íntegra e de forma on-line.

\section{Discussão}

\subsection{Possíveis causas da diarréia em crianças de 2 a 6 anos}

\subsubsection{Condições sociais}

A doença diarreica ainda persiste como um problema de saúde pública, sendo uma das principais causas de mortalidade infantil nos países subdesenvolvidos, salientando-se que tais óbitos são considerados um indicador de situações de pobreza. 
Segundo esse alto índice decorre do peso relativo dos óbitos por diarréias, pneumonias e desnutrição, responsáveis pela maior parte das mortes infantis tardias (Szwarcwald et al., 1992).

Porém, o baixo nível econômico social, a falta de saneamento e o comportamento higiênico estão relacionados à morbimortalidade das crianças. No Brasil, o indicador de extrema pobreza destacou-se em todas as regiões do país exceto na região Sul. No Norte e Nordeste o óbito por diarréia se destaca devido às condições sociais e demográficas e ao saneamento básico. Já nas microrregiões Centro-Oeste e Sudeste os indicadores mostraram serem significativos com os óbitos por diarréia infantil, pois nas mesmas há uma diferença econômica importante, comparando com a região nordeste, que é muito pobre aumentando assim os índices de insalubridade (Buhler et al., 2014).

Diversos estudos têm mostrado uma associação entre a ocorrência de diarréia e a baixa renda das famílias, falta de água, lixo ambiental, fatores estes que estão presentes diariamente em regiões de periferias, favelas e cortiços (Santos, Martins \& Sawaya, 2008). Algumas intercorrências infecciosas, principalmente diarréia e infecções respiratórias estão relacionadas à desnutrição, e quanto maior a frequência desses episódios, maior é a gravidade e maior o efeito sobre o estado nutricional da criança, tendo em vista que no Brasil quando não há recursos suficientes, para compra de alimentos nutritivos estes acabam não chegando até a mesa dos brasileiros menos favorecidos, o que consequentemente levam a um estado de desnutrição maior. (Vanderlei, Silva \& Braga, 2003).

Sabe-se também que essa falta de recursos ocasiona a aceitação de alimentos e bebidas de alimentos advindos de doações o que nem sempre é sinônimo de alimento ou bebida nutritivo sendo muitas vezes, a única fonte energética de alimentação. O que pode consequentemente ocasionar em diarreia. (Santos, Martins \& Sawaya, 2008)

\subsubsection{Intoxicação alimentar e Agentes patogênicos}

O Brasil é um dos países mais atingidos por doenças diarréicas, afetando diretamente as taxas de mortalidade infantil. Anualmente, falecem 50 mil crianças menores de um ano em consequência da diarreia. Fatores como desmame precoce, contaminação dos alimentos e água potável e a desnutrição são favoráveis ao aparecimento da diarréia aguda (Campos et al., 1995).

Segundo o Manual de Boas Práticas de Manipulação de Alimentos (Brasil, 2015), considerando que as cozinhas das escolas são caracterizadas como Serviços de Alimentação Coletiva, é necessário garantir que os alimentos produzidos ofereçam segurança e qualidade sanitária aos escolares e atendam a legislação sanitária em vigor, minimizando risco de ocorrência de Doenças Transmitidas por Alimentos (DTA) nos escolares.

A capacitação de manipuladores de alimentos tornou-se uma necessidade presente em todos os setores que envolvem a alimentação, particularmente em unidades de alimentação de centros de educação infantil, popularmente conhecidos como creches. Pelo fato de estarem diariamente em contato com crianças e serem responsáveis pela segurança da alimentação delas, é de fundamental importância que seus conhecimentos sejam constantemente reciclados, porém, nem sempre o que é trazido pela teoria é o que se pode ver na prática. (Silva \& Dantas, 2017).

De acordo com Costa et al. (2018) o estado nutricional de cada criança é de extrema importância, o conhecimento de uma boa alimentação ajuda em bons hábitos alimentares, em uma educação alimentar correta e bem manuseada evitando futuras ações de promoção de saúde. Advindas de patógenos que podem ser fatais como: Salmonella, Shigella, Escherichia coli, Ameba, Giárdia lamblia e dietéticas: como intolerâncias alimentares, além de outros fatores.

São diversos os microrganismos patogênicos de origem alimentar, presentes nas mãos dos manipuladores, nos alimentos, água e no ambiente exposto, destacam-se: bactérias (E. coli, E.coli enterohemorrágica, E.coli enterotoxigênica, E.coli enteropatogênica, E.coli enteroinvasiva, E.coli enteroagregativa, Campylobacter, Shigella, Víbrium cholerae, Yersínia, 
Clostridium difficile, Salmonella), vírus (Rotavírus e os Norovírus) e parasitas (nematódeos, cestódeos, trematódeos e protozoários) (Teixeira, 2010).

A adesão dos microrganismos à mucosa do intestino humano, com posterior proliferação é a principal origem das infecções alimentares, sendo que $E$. coli possui destaque entre as bactérias invasivas e comumente ocasiona diarréias constantes com sangue e pus, dores abdominais intensas, febre e desidratação leve (Maldotti \& Dalzochio, 2021).

\subsubsection{Falta de saneamento básico}

A ausência de saneamento básico ainda é um problema que atinge boa parte da população brasileira, principalmente nas áreas periurbanas do país (Paz, Almeida \& Günther, 2012). Dentre os problemas causados pela falta de saneamento básico, a doença diarreica nas crianças é uma síndrome frequente, estando entre as principais causas de consulta, internação e mortalidade, sobretudo em países em desenvolvimento (Pontual, Falbo \& Gouveia, 2006).

A morbidade por diarréia infantil está condicionada principalmente ao baixo nível socioeconômico da população, sendo este um dos principais fatores que influencia as condições de saneamento básico precário e comportamento higiênico pessoal e doméstico insatisfatório (Buhler et al., 2014).

Portanto, faz-se necessário buscar e aplicar certas medidas evitando o agravo da diarréia, principalmente em relação ao saneamento básico, visto que ele é uma das principais fontes de contaminação dessa doença. Neste sentido, políticas públicas sociais, econômicas, ambientais, culturais e de saúde devem embasar-se no princípio de equidade para atender as diferentes necessidades locais de cada região (Buhler et al., 2014).

\subsubsection{Ausência de imunização}

Segundo Oliveira e Linhares (1999), a infecção por Rotavírus é a causa mais comum de diarréia infantil grave em todo o mundo, podendo culminar em desidratação e até ao óbito. Sua importância reflete em aproximadamente 600 mil óbitos, 2 milhões de hospitalizações, 25 milhões de atendimentos de saúde e 111 milhões de episódios de diarréia em crianças jovens a cada ano no mundo todo (Ambrosini \& Carraro, 2012).

Mesmo com inúmeras campanhas de vacinação, ainda encontram-se pessoas "anti-vacinas", ou que até mesmo não respeitam a importância e necessidade de vacinar seus filhos. Pois, ainda existem casos que poderiam ser evitados com a vacina, dentre eles o rotavírus onde a sua administração é recomendada com (2 e 4 meses de vida), juntamente com as vacinas de S. pneumoniae para evitar o esquecimento.

Levantamentos epidemiológicos em escala mundial observaram a incidência de rotavírus entre 12 e $71 \%$, com média de 34\% em crianças com menos de três anos de idade com diarréia aguda. No Brasil, a incidência de rotavírus nas crianças com gastrenterite atendidas em ambulatórios ou hospitais, é da ordem de 12 a 42\%, essas porcentagens merecem atenção, em especial pois a faixa etária mais acometida é a pré-escolar onde subentende-se que é da responsabilidade dos pais ou responsáveis, garantir a vacinação correta das crianças (Silva et. al., 2010).

Em 2006 houve uma atualização e adequação do Calendário Básico de Vacinação da Criança, com a introdução da vacina oral contra o rotavírus humano (VORH), mantendo-se as demais vacinas para este calendário e para o Calendário de Vacinação do Adolescente e o Calendário de Vacinação do Adulto e Idoso, publicados em 2004. Analisando as coberturas vacinais verificou-se que as vacinas BCG e rotavírus apresentaram as mais baixas coberturas vacinais, independente da obrigação, ainda existem casos de diarreia por conta do rotavírus em todo o país. (Escórcio, 2014). 


\subsubsection{Alergias e intolerâncias alimentares}

A prevalência da doença alérgica tem aumentado drasticamente nas últimas décadas, sobretudo em idade pediátrica. Vários dos alérgenos alimentares mais comuns são aqueles que compreendem a maior porção dos nutrientes das dietas de crianças em desenvolvimento. Vale salientar que outros fatores limitantes das condições de saúde concorrem com a alergia alimentar, em especial nas populações de baixas condições socioeconômicas, como outras restrições e impropriedades alimentares conjugadas com o estresse das doenças, sobretudo de natureza infecciosa. Não é incomum, entretanto, familiares de crianças com alergia alimentar encontrarem dificuldades quanto à obtenção de subsídios alocados pelo poder público para a compra de fórmulas específicas para o consumo (Correia, 2020).

Se compreende como alergia alimentar um efeito adverso de saúde que ocorre na sequência de uma resposta imune específica e reprodutível de acordo com a exposição ao alimento. Este conceito é distinto da intolerância alimentar que envolve reações não-imunes, nomeadamente metabólicas, tóxicas, farmacológicas e outras com mecanismos inespecíficos. Embora haja mais de 170 alimentos descritos como potencialmente alergênicos, a maioria das reações são desencadeadas por um número restrito de alimentos, havendo variações geográficas entre regiões. A anafilaxia induzida por alimentos é uma reação alérgica grave, de início rápido, que pode em último caso conduzir à morte e é a causa mais frequente de anafilaxia em idade pediátrica (Marques, 2015).

Uma implementação de estratégias de prevenção e tratamento dirigidas a esta problemática, são cruciais para melhorar a qualidade de vida destas crianças e suas famílias, onde os profissionais que trabalham nas escolas em articulação com os profissionais de saúde, assumem um papel determinante. As estratégias de intervenção identificadas com resultados mais efetivos, foram a formação e treino dos profissionais que trabalham nas escolas, o tratamento de reações alérgicas e a adoção de políticas escolares de redução do risco de exposição a alergênicos (Pinheiro, Fidalgo \& Mendes, 2020).

\subsection{Consequências da diarréia}

Uma série de estudos documentam que as doenças entéricas na primeira infância estão associadas a efeitos profundos a longo prazo no desenvolvimento físico e cognitivo posterior, bem como ao crescimento físico (Guerrant, et al., 1999).

As consequências não são apenas imediatas, segundo Patrick, et al., (2005), os efeitos de muitos episódios de diarréia nos primeiros anos de vida, prejudicam áreas primordiais como a fluência verbal, pois a desidratação advinda de uma diarreia intensa como de uma giardia, leva a criança a um cansaço que não o deixa motivado a se esforçar mentalmente e fisicamente, além da má nutrição. Ainda na primeira infância, podem, também afetar o desenvolvimento neuropsicológico inicial, a déficits de longo prazo no desenvolvimento cognitivo normal. Avaliando como base que até os 2 anos de idade a formação neuronal junto com o desenvolvimento cognitivo tem sua maturação diretamente influenciada por fatores ambientais.

Embora doenças diarreicas sejam bem reconhecidas, o impacto da morbidade de longo prazo de numerosas doenças diarreicas não fatais desidratantes e desnutritivas repetidas ou prolongadas em áreas empobrecidas nos primeiros anos de formação crítica sobre o crescimento e desenvolvimento infantil está sendo abordado apenas recentemente (Lorntz et al,. 2006).

\section{Considerações Finais}

Conforme os objetivos propostos neste trabalho, e avaliar as causas e também possíveis consequências da diarreia em crianças de idade pré escolar, pode-se concluir que foi possível, através da busca - entre artigos, livros, sites de cunho governamental, dentre outros trabalhos que contém informações científicas - verificar que as principais causas estão relacionadas com: condições sociais, agentes patogênicos, falhas na imunização e intolerâncias e alergias alimentares. 
Tendo como principais consequências, desidratação intensa, desnutrição, internação em casos graves, déficits em seu desenvolvimento físico e cognitivo quando não diagnosticado corretamente, podendo até mesmo levar a óbito.

Reitera-se, então, a importância da atenção e dos cuidados, tanto dos pais quanto dos responsáveis, pois são os principais responsáveis por administrar o que crianças comem, bebem, como se relacionam, medicamentos que utilizam e todos os requisitos mínimos necessários para que a saúde se mantenha com qualidade. Sendo assim, recomenda-se a garantia de alimentos nutritivos, bem higienizados, exigir saneamento básico adequado, manter sempre o cartão vacinal em dia, além de consultas recomendadas por profissionais da saúde.

Pode-se verificar que, sem os requisitos mínimos citados nesse trabalho, não há como garantir o bem-estar e total segurança das crianças, a fim de evitar não somente a diarréia aguda, como outras doenças relacionadas às causas citadas.

Como sugestão para trabalhos futuros, acredita-se que o estudo sobre as possíveis causas de diarreia em crianças préescolares, seja relevante, pois ainda é uma realidade enfrentada no país, em especial em regiões menos favorecidas, tanto economicamente e socialmente. A busca por novos estudos deve ser periódica, para avaliar as melhorias de condições e o número de casos de diarreia nessas regiões.

\section{Referências}

Aguiar, K. C. G. D., Cohen, S. C., Maciel, E. M. G. D. S. \& Kligerman, D. C. (2020). Fatores de risco para ocorrência de diarréia em crianças residentes na Ilha de Guaratiba (RJ). Saúde em Debate, 44, 205-220.

Ambrosini, V. A., \& Carraro, E. (2012). Impacto da vacinação contra rotavírus no Brasil. Medicina (Ribeirão Preto), 45(4), 411-418.

Brasil.2015. Boas Práticas de Manipulação de Alimentos. http://www.gestaoescolar.diaadia.pr.gov.br/arquivos/File/alimenatacao_escolar/ manual_de_boas_praticas_2016.pdf.

Brasil. 2021. Doenças diarreicas agudas (DDA): causas, sinais e sintomas, tratamento e prevenção. https://bit.ly/3oSmIv1.

Bühler, H. F., Ignotti, E., Neves, S. M. A. D. S., \& Hacon, S. S. (2014). Análise espacial de indicadores integrados determinantes da mortalidade por diarréia aguda em crianças menores de 1 ano em regiões geográficas. Ciência \& Saúde Coletiva, 19, 4131-4140.

Campos, G. D. J. D. V., Reis Filho, S. A. D., Silva, A. A. M. D., Novochadlo, M. A. D. S., Silva, R. A. D., \& Galvão, C. E. (1995). Morbimortalidade infantil por diarréia aguda em área metropolitana da região Nordeste do Brasil, 1986-1989. Revista de Saúde Pública, 29, $132-139$.

Correia, J. A. D. S. (2020). Prevalência de alergia alimentar em pré-escolares de Limoeiro-PE (Master's thesis, Universidade Federal de Pernambuco).

Costa, M. C., de Sousa, A. F., do Nascimento Lima, J. T., de Sousa, S. D. F., Ferreira, F. V., \& de Azevedo Marques, A. R. (2018). Estado nutricional, práticas alimentares e conhecimentos em nutrição de escolares. Revista de Atenção à Saúde (ISSN 2359-4330), 16(56), $12-17$.

Escórcio, I. C. D. C. (2017). Cobertura vacinal em menores de um ano de 2008 a 2012 no município de Timon-MA e planejamento de atividade educativa junto à equipe de enfermagem e pais para discussão e atualização sobre o tema.

Estrela, C. (2018). Metodologia científica: ciência, ensino, pesquisa. Artes Médicas.

Gandra, Y. R. (1981). O pré-escolar de dois a seis anos de idade e o seu atendimento. Revista de Saúde Pública, 15, 3-8.

Guerrant, DI, Moore, SR, Lima, AA, Patrick, PD, Schorling, JB e Guerrant, RL (1999). Associação de diarréia infantil e criptosporidiose com comprometimento da aptidão física e função cognitiva quatro a sete anos depois em uma comunidade urbana pobre no nordeste do Brasil. The American Journal of Tropical Medicine and Hygiene, 61 (5), 707-713.

Hayakawa, A. P. S., Sadowski, R. A., \& Fernandes, M. I. M. (2010). Histórico sobre a vacina contra o Rotavírus. Revista Uningá Review, 1(1).

Kleina, C., \& Rodrigues, K. S. B. (2014). Metodologia da pesquisa e do trabalho científico. Ed. Curitiba, PR: IESDE Brasil.

Linhares, A. C., \& Villa, L. L. (2006). Vacinas contra rotavírus e papilomavírus humano (HPV). Jornal de Pediatria, 82, s25-s34.

Lorntz, B., Soares, A. M., Moore, S. R., Pinkerton, R., Gansneder, B., Bovbjerg, V. E., ... \& Guerrant, R. L. (2006). Early childhood diarrhea predicts impaired school performance. The Pediatric infectious disease journal, 25(6), 513-520.

Maldotti, J., \& Dalzochio, T. (2021). Parasitos intestinais em crianças no Brasil: Revisão Sistemática. Revista Cereus, 13(1), 62-73.

Marques, J. G. (2015). Alergia alimentar em crianças em idade pré-escolar (Doctoral dissertation).

Moraes, A. C., \& Castro, F. M. (2014). Diarréia aguda. Journal of Moleular Biology, 102, 21-28. 
Research, Society and Development, v. 10, n. 13, e575101319760, 2021

(CC BY 4.0) | ISSN 2525-3409 | DOI: http://dx.doi.org/10.33448/rsd-v10i13.19760

Oliveira, C. S. D., \& Linhares, A. D. C. (1999). Rotavírus: Aspectos clínicos e prevenção.

Patrick, P. D., Oriá, R. B., Madhavan, V., Pinkerton, R. C., Lorntz, B., Lima, A. A., \& Guerrant, R. L. (2005). Limitations in verbal fluency following heavy burdens of early childhood diarrhea in Brazilian shantytown children. Child Neuropsychology, 11(3), $233-244$.

Paz, M. G. A. D., Almeida, M. F. D., \& Günther, W. M. R. (2012). Prevalência de diarréia em crianças e condições de saneamento e moradia em áreas periurbanas de Guarulhos, SP. Revista Brasileira de Epidemiologia, 15, 188-197.

Pinheiro, A. C., Fidalgo, C., \& Mendes, C. M. F. G. D. S. (2020). Estratégias de intervenção em ambiente escolar dirigida às crianças com alergia alimentar. Salutis Scientia-Revista de Ciências da Saúde da ESSCVP, 12, 18-24.

Pontual, J. P. D. S., Falbo, A. R., \& Gouveia, J. D. S. (2006). Estudo etiológico da diarréia em crianças hospitalizadas no Instituto Materno Infantil Prof. Fernando Figueira, IMIP, em Recife, Pernambuco. Revista Brasileira de Saúde Materno Infantil, 6, s11-s17.

Prodanov, C. C., \& De Freitas, E. C. (2013). Metodologia do trabalho científico: métodos e técnicas da pesquisa e do trabalho acadêmico-2a Edição. Editora Feevale.

Santos, R. B., Martins, P. A., \& Sawaya, A. L. (2008). Estado nutricional, condições socioeconômicas, ambientais e de saúde de crianças moradoras em cortiços e favela. Revista de Nutrição, 21, 671-681.

Silva, A. S. J., \& Dantas, A. C. D. M. (2017). Capacitação em boas práticas para merendeiras e auxiliares que atuam na educação infantil. In VII Salão integrado de ensino, pesquisa e extensão, III Jornada de Pós-graduação e II Seminário sobre Territorialidade.

Silva, M. L. D., Souza, J. R. D., \& Melo, M. M. M. D. (2010). Prevalência de rotavírus em crianças atendidas na rede pública de saúde do estado de Pernambuco. Revista da Sociedade Brasileira de Medicina Tropical, 43, 548-551.

Sociedade Brasileira de Pediatria - SBP. 2021. Diarréias. https://bit.ly/3fh9iFD.

Soragni, L., Barnabe, A. S., \& de Campos Mello, T. R. (2019). Doenças transmitidas por alimentos e participação da manipulação inadequada para sua ocorrência: uma revisão. Estação Científica (UNIFAP), 9(2), 19-31.

Szwarcwald, C. L., Leal, M. D. C., Castilho, E. A. D., \& Andrade, C. L. (1997). Mortalidade infantil no Brasil: Belíndia ou Bulgária?.Cadernos de Saúde Pública, $13,503-516$.

Teixeira, A. F. M. (2010). Doenças microbianas de origem alimentar. Academia de Ciência e Tecnologia, 3, 1-8.

Vanderlei, L. C. D. M., Silva, G. A. P. D., \& Braga, J. U. (2003). Fatores de risco para internamento por diarréia aguda em menores de dois anos: estudo de caso-controle. Cadernos de saúde pública, 19, 455-463.

Victora, C. G. (2009). Mortalidade por diarréia: o que o mundo pode aprender com o Brasil?. Jornal de Pediatria, 85(1), 3-5. 